\title{
Transcriptome sequencing and anthocyanin metabolite analysis determines leaf color of Acer pseudo-sieboldianum in autumn
}

Donghui Zhao

Yanbian University

\section{Yufu Gao}

Yanbian University

Liping Rong ( $\square$ rongliping2013@163.com )

Yanbian University

\section{Research article}

Keywords: Acer pseudo-sieboldianum, Transcriptome, Differentially expressed genes, Anthocyanin

Posted Date: December 6th, 2019

DOl: https://doi.org/10.21203/rs.2.18312/v1

License: (c) (i) This work is licensed under a Creative Commons Attribution 4.0 International License.

Read Full License 
1 Transcriptome sequencing and anthocyanin

2 metabolite analysis determines leaf color of Acer

3 pseudo-sieboldianum in autumn

4 Donghui Zhao ${ }^{1}$, Yufu Gao ${ }^{1}$, Liping Rong ${ }^{1 *}$, Jiaqi Zhang ${ }^{1}$ and Jiashuo Chen ${ }^{1}$

5 Abstract

Background: Acer pseudo-sieboldianum is an often used color-leafed tree native to northeastern China, whose leaves are gorgeous in autumn and is used to great effect in landscaping. Leaf color is an important index affecting the ornamental traits of color-leafed plants. Thus, it is of great significance to study the mechanism in leaf coloring in Acer pseudo-sieboldianum as the development and application prospects of this research are broad, and further the utilization of local characteristics tree species with colored leaves.

Results: In this study, the leaves of Acer pseudo-sieboldianum from three stages in the autumn color-changing period were used as materials. In order to investigate the related differentially expressed genes in the process of color transformation, we determined the anthocyanins in leaves and used high-throughput sequencing to analyze the associated transcriptomes. The results demonstrated that five anthocyanins were detected in this experiment. It is worth mentioning that Peonidin O-hexoside, Cyanidin 3-O-glucoside (Kuromanin) and Cyanidin 3,5-O-diglucoside (Cyanin) have significant differences in their content change in two of the tested periods, meaning these may be the key substances determining the final color of Acer pseudo-sieboldianum. A total of $67.47 \mathrm{~Gb}$ of clean data were obtained from our sequencing results. Functional annotation of unigenes, including comparison with COG and GO databases, yielded 35,316 unigene annotations. 16521 differentially expressed genes were identified from a statistical analysis of differentially gene expression. We were able to cluster genes related to leaf

\footnotetext{
* Correspondence: rongliping2013@163.com

${ }^{1}$ Agriculture college, Yanbian University, Yanji 133002, China

Full list of author information is available at the end of the article
} 
color formation in Acer pseudo-sieboldianum, including ANS, DFR, F3H, UFGT, and so on, based on the expression at specific times.

Conclusions: This study enriched the available transcriptome information for Acer pseudo-sieboldianum and identified a series of differentially expressed genes related to leaf color, which provides valuable information for further study on the genetic mechanism of leaf color expression in Acer pseudo-sieboldianum.

Key words: Acer pseudo-sieboldianum, Transcriptome, Differentially expressed genes, Anthocyanin

\section{Background}

Ornamental plants are widely used in landscape greening and green plant allocation. Leaf color and flower color are one of the most important characteristics of ornamental plants $[1,2]$. Some scholars have systematically analyzed and determined the pigments and physiological indexes of the leaves of color-leafed plants [3-5]. Research shows that the change of leaf color is closely related to the synthesis and accumulation of flavonoids and anthocyanins in leaves[6]. Flavonoids are one of the important secondary metabolites of plants and they often have anti-cancer, anti-oxidation and anti-atherosclerosis properties [7]. Flavonoids can be classified into flavanone, isoflavone, flavone, flavonol, dihydroflavone and anthocyanin depending on their different structures[8]. In recent years, some scholars have identified PAL, CHS, CHI, DFR, ANS, F3H, F3'H, F3'5'H [9-12] and a few related regulatory genes such as MYB, bHLH and WDR, in color changing leafed plants[13, 14]. However, the process of anthocyanin synthesis and accumulation is relatively complex, and is regulated by multiple enzymes and transcription factors[15], as well as being influenced by external environmental factors such as light[16], water stress[17], temperature[18] and other factors. Thus, the mechanism of leaf color change in color-leaved plants needs to be further studied.

Acer pseudo-sieboldianum, belongs to the Acer genus of the family Sapindaceae. It is a small deciduous tree, and because of its beautiful shape and brilliant leaves, it is an often used autumn leaf ornamental tree species [19]. It is a type of nectar plant, whose woods can be used for making utensils and leaves can be used as dyes, giving it high 
economic value [20]. Recently, some scholars have reported and studied the introduction, cultivation and breeding of Acer pseudo-sieboldianum [21, 22]. However, the key genes affecting leaf color change in Acer pseudo-sieboldianum have not been determined yet, and genome information on Acer pseudo-sieboldianum is relatively scarce. This fact means that the molecular regulatory mechanisms related to leaf color formation needs further study.

In recent years, transcriptome high-throughput sequencing technology has been widely used to study the mechanism of leaf color in various plants [23, 24]. In this study, we performed transcriptome sequencing, assembly, annotation and bioinformatic analysis on leaves from Acer pseudo-sieboldianum at different color-changing stages in autumn. We then combined this data with anthocyanin metabolism analysis data to identify some enzymes related to flavonoid and anthocyanin synthesis, and using real-time fluorescence quantitative qPCR, we analyzed and validated these DEGs in the leaves of Acer pseudo-sieboldianum at different transformation stages in autumn. We thus screened to identify the candidate genes affecting leaf color in Acer pseudo-sieboldianum, which provides a theoretical basis for studying the molecular mechanism of leaf color in Acer pseudo-sieboldianum.

\section{Materials and methods}

\section{Plant materials and treatments}

The materials tested were Acer pseudo-sieboldianum plants that were 5 years old from Tianchi Square, Yanji City, Jilin Province. Samples from the same Acer pseudo-sieboldianum plant were collected separately and storing in a refrigerator at $-80^{\circ} \mathrm{C}$ for transcriptome sequencing and anthocyanin metabolite analysis from three periods on September 21, 2018 (B), September 30, 2018 (M) and October 11, 2018 (A). Harvest was repeated three times per treatment facility (Fig. 1). 


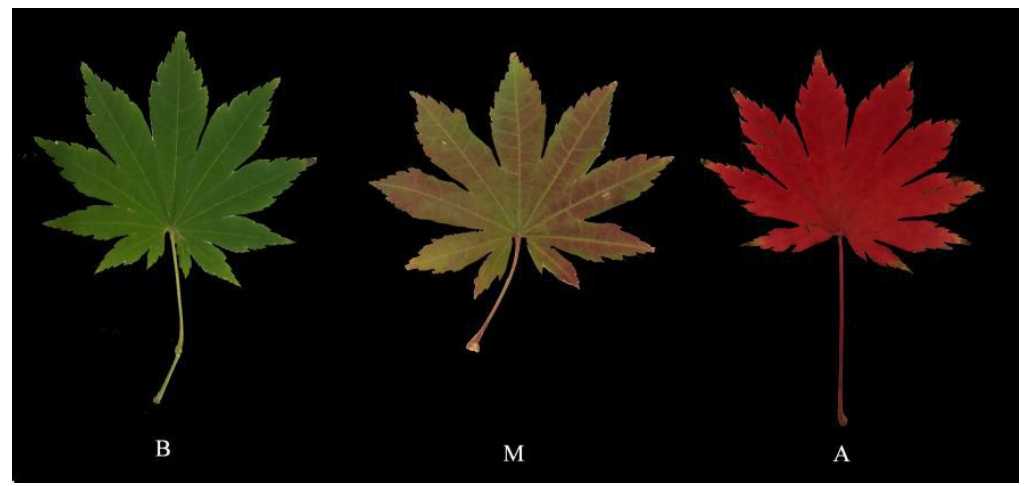

Fig. 1 The color change of leaf transcriptome level of Acer pseudo-sieboldianum at different periods in autumn can be divided into three stages. Initial stage: at this early stage (B), the leaves were all green. Mid stage: at this time, the leaves were both red and green (M). The last stage: the leaves were all red.

\section{Extraction, identification and data analysis of anthocyanin metabolites}

Leaf tissue samples of Acer pseudo-sieboldianum were ground to a powder, and $100 \mathrm{mg}$ of powder was dissolved in $1.0 \mathrm{ml}$ extract solution (70\% methanol aqueous solution). The dissolved sample was placed in a refrigerator overnight at $4{ }^{\circ} \mathrm{C}$, and then vortexed three times during the period to improve the extraction rate. After centrifugation, the supernatant was reserved, the sample was filtered with a microporous filter membrane, and stored in a sample bottle for LC-MS / MS analysis. We used multiple reaction monitoring (MRM) for qualitative and quantitative analysis of metabolites by mass spectrometry. Combining single variable statistical analysis and multivariate statistical analysis, we calculated the fold-change, and called a metabolite as a differential metabolite when its values was between a fold change $\geq 2$ and a fold change $\leq 0.5$. The differential metabolites were then annotated using the KEGG database[25].

\section{RNA isolation, library construction and RNA-Seq}

Total RNA was extracted from leaf samples using an RNA extraction kit (Beijing Tiangen in China). Agarose electrophoresis and the Agilent 2100 Bioanalyzer were used to determine the concentration, purity and integrity of RNA samples. Then, PolyA mRNA was reverse transcribed into cDNA, and the construction and sequencing of the cDNA library was completed by the BMK Technology company in Beijing. Raw reads were obtained using an Illumina Hiseq 2500 sequencing platform, and after filtering, clean 
reads were obtained. Contigs were assembled by overlapping information between sequences, transcripts were locally assembled, and unigenes were obtained by homologous clustering and splicing of transcripts with Tgicl and Phrap software, respectively[26].

\section{De novo assembly and functional annotation}

After obtaining high quality sequencing data, it was necessary to assemble the genomic sequence of Acer pseudo-sieboldianum. First, Trinity software parsed the sequencing reads into shorter fragments (K-mers), extends these fragments into longer fragments (Contig), and uses the overlap between these fragments to determine the fragment set (Component). Finally, using the dual methods of De Bruijn mapping and sequencing read information analysis, each transcript sequence was identified in each fragment set. The Unigene sequence was compared with the gene sequence in NR [27], Swiss-Prot [28], GO [29], COG[30], KOG [31], eggNOG4.5 [32], KEGG database by Blast software [33] (e $<0.00001$ ). Using KOBAS 2.0 [34], the KEGG orthology result of unigenes from KEGG was obtained, and after predicting the amino acid sequence of each unigene, we used HMMER [35] software to compare with the Pfam [36] database, select unigenes whose BLAST parameter E-values were not greater than $1 \mathrm{e}^{-5}$ and whose HMMER parameter E-values were not more than $1 \mathrm{e}^{-10}$, and thus, finally obtained a unigene with annotation information.

\section{Expression and differentially expressed unigene annotation}

Bowtie[37] was used to compare the sequenced reads with a unigene library, and RSEM[38] was used to estimate the expression level. The expression abundance of each corresponding unigene was expressed by its FPKM [39] value. It is a common method for estimating gene expression level in transcriptome sequencing data analysis. The use of FPKM values can eliminate the influence of gene length and sequencing on calculations of gene expression. When detecting differentially expressed genes, DESeq2 was used to analyze the differentially expressed genes between the sample groups, and the differentially expressed gene sets between two different conditions were identified. In the process of differential expression analysis, the Benjamini-Hochberg method was 
used to correct the significance p-value of the original hypothesis test, so as to reduce the false positives in independent statistical hypothesis testing for a large number of gene expression values. In the screening process, the criterion was that the FDR (False Discovery Rate) was less than 0.01 and the difference factor FC (Fold Change) was greater than or equal to 2. Between these two factors, the FC represented the ratio of expression between two samples (groups).

\section{Gene validation and expression analysis}

In order to validate our differential gene expression analysis, RT-qPCR was used to validate the differentially expressed genes related to flavonoid anthocyanin biosynthesis[40]. We used a fluorescence quantitative Kit $\left(2 \times\right.$ SYBR ${ }^{\circledR}$ green premix $)$ and an analytikjena-qTOWER2.2 fluorescence quantitative PCR instrument for quantitative analysis. The primer sequences can be found in File. S1. The reaction procedure was as follows: $95^{\circ} \mathrm{C}$ for $3 \mathrm{~min}, 95^{\circ} \mathrm{C}$ for $10 \mathrm{~s}, 58^{\circ} \mathrm{C}$ for $30 \mathrm{~s}$, for a total of 39 cycles. Melt curve analysis $\left(60{ }^{\circ} \mathrm{C} \sim 95{ }^{\circ} \mathrm{C},+1{ }^{\circ} \mathrm{C} /\right.$ cycle, holding time $\left.4 \mathrm{~s}\right)$, and carried out centrifugation on PCR plate centrifuge at $4^{\circ} \mathrm{C} 6000 \mathrm{rpm}$ for $30 \mathrm{~s}$. Finally, we put it in quantitative PCR for amplification, using c110191.graph_c0 as an internal reference gene. The procedure was repeated three times for each sample, and we calculated the relative gene expression of each sample using the $2^{\wedge}-\triangle \triangle \mathrm{Ct}$ method.

\section{Results}

Contents of flavonoid-anthocyanin biosynthesis in the leaves of Acer pseudo-sieboldianum

In order to further understand the mechanism of pigment formation in Acer pseudo-sieboldianum leaves, we carried out qualitative analysis of anthocyanin components in the middle and last stage of leaf color transformation. According to our UPLC-Q-TOF-MS data, five anthocyanins were identified (Table 1). These were Peonidin O-hexoside, Rosinidin O-hexoside, Cyanidin 3-O-glucoside (Kuromanin), Cyanidin 3,5-O-diglucoside (Cyanin) and Pelargonidin 3-O-beta-D-glucoside (Callistephin chloride). 
The content changes of these five metabolites were different during the middle and last stages of color transformation. Rosinidin O-hexoside and Pelargonidin

163 3-O-beta-D-glucoside (Callistephin chloride) anthocyanin content showed little 164 difference between these two periods. But Peonidin O-hexoside, Cyanidin 3-O-glucoside (Kuromanin) and Cyanidin 3,5-O-diglucoside (Cyanin) displayed significant differences in content change between these two periods, meaning they may be the key substances for the final color of Acer pseudo-sieboldianum (File. S2).

Table1 Anthocyanins detected in Acer pseudo-sieboldianum

\begin{tabular}{ccccc}
\hline & & & Average & Average \\
Index & KEGG & Compounds & content in M & content in A \\
& ID & & stage & stage \\
\hline Pma1590 & - & Peonidin O-hexoside & 1425333 & 26682600 \\
Pmb0545 & - & Rosinidin O-hexoside & 48403 & 27156 \\
Pmb0550 & C08604 & Cyanidin3-O-glucoside & 2420000 & 6970767 \\
Pme1777 & C08639 & Cyanidin 3,5-O-diglucoside & 14336000 & 70564000 \\
Pme3392 & - & Pelargonidin 3-O-beta-D-glucoside & 42512 & 62136 \\
\hline
\end{tabular}

169

170

171

RNA sequencing and de novo assembly of the Acer pseudo-sieboldianum genome

In order to understand the molecular mechanism of color change in Acer pseudo-sieboldianum leaves in autumn, nine libraries (early stage: B1, B2, and B3, medium stage: M1, M2, and M3, last stage: A1, A2, and A3) were constructed from leaves from the three different stages, with three replicate libraries being constructed per stage. Sequencing was performed using the Illumina Hiseq 2500, and a total of $67.47 \mathrm{~Gb}$ of clean data was obtained from these sequencing results. The clean data from all samples totally $6.14 \mathrm{~Gb}$, and the percentage of Q30 bases was $93.10 \%$ or more. After assembly, 50,501 unigenes were identified. Among these, there were 20,706 unigenes over $1 \mathrm{~kb}$ in length, and the error rate of sequencing was less than $0.1 \%$, which indicates that the quality of sequencing data was good and could be used for subsequent analysis (Table 2). 
182 These recombinant sequence dataset yielded 115,413 transcripts and 50,501 unigenes,

183 among which, the N50 (accounting for 50\% of the maximum length nucleotide sequence

184 of all single genes) was $2267 \mathrm{nt}$ and $1979 \mathrm{nt}$, respectively. There were 17,366 (34.39\%)

185 unigenes between 300 and $500 \mathrm{nt}, 23,580$ (46.69\%) unigenes between 500 and $2000 \mathrm{nt}$, 186 and 9,555 (18.92\%) unigenes longer than $2000 \mathrm{nt}$ (Table 3).

187 Table 2 Statistics of sequencing data across the nine libraries in Acer 188 pseudo-sieboldianum

\begin{tabular}{lllllll}
\hline \multicolumn{2}{l}{ Sample II Clean } & Base number & GC contmer $\% \geq Q 30$ & Mapped reads & Mapped ratio \\
number & & & & & \\
\hline B1 & $24,891,319$ & $7,411,181,104$ & $45.12 \%$ & $94.12 \%$ & $20,710,424$ & $83.20 \%$ \\
B2 & $24,754,322$ & $7,373,450,408$ & $45.72 \%$ & $94.25 \%$ & $20,509,397$ & $82.85 \%$ \\
B3 & $26,344,753$ & $7,839,350,686$ & $44.02 \%$ & $93.10 \%$ & $21,123,549$ & $80.18 \%$ \\
M1 & $20,586,684$ & $6,140,849,238$ & $44.61 \%$ & $94.04 \%$ & $17,004,609$ & $82.60 \%$ \\
M2 & $24,264,520$ & $7,220,440,952$ & $45.08 \%$ & $94.33 \%$ & $20,030,805$ & $82.55 \%$ \\
M3 & $20,951,279$ & $6,256,334,358$ & $44.34 \%$ & $93.99 \%$ & $17,134,598$ & $81.78 \%$ \\
A1 & $26,611,490$ & $7,923,701,850$ & $44.84 \%$ & $94.03 \%$ & $21,802,156$ & $81.93 \%$ \\
& & & & & & \\
A2 & $28,672,411$ & $8,529,990,156$ & $44.95 \%$ & $93.73 \%$ & $23,549,563$ & $82.13 \%$ \\
A3 & $29,433,309$ & $8,770,181,170$ & $45.13 \%$ & $93.44 \%$ & $24,196,882$ & $82.21 \%$ \\
\hline
\end{tabular}

\begin{tabular}{lll}
\hline \multicolumn{3}{l}{ Table 3 Length distributions of the transcripts and unigenes from de novo assembly } \\
\hline Length range & Transcript & Unigene \\
\hline $300-500$ & $24,236(21.00 \%)$ & $17,366(34.39 \%)$ \\
$500-1000$ & $26,476(22.94 \%)$ & $12,429(24.61 \%)$ \\
$1000-2000$ & $33,114(28.69 \%)$ & $11,151(22.08 \%)$ \\
$2000+$ & $31,587(27.37 \%)$ & $9,555(18.92 \%)$ \\
Total Number & 115,413 & 50,501 \\
Total Length & $179,159,431$ & $62,348,493$ \\
N50 Length & 2,267 & 1,979 \\
Mean Length & 1552.33 & 1234.60 \\
\hline
\end{tabular}




\section{Functional annotation and classification}

Unigene sequence was then compared with gene sequences in the NR, Swiss-Prot, GO, COG, KOG, eggNOG4.5, and KEGG databases using BLAST software (e < 0.00001). 35,316 unigenes were identified, accounting for $70.01 \%$ of the 50,501 unigenes. 12,984 unigenes were annotated in the COG database, 25,375, 12,487 and 19,460 unigenes were annotated in the GO, KEGG and KOG databases, respectively. 25,226 unigenes were annotated in the Pfam database. 19,796 unigenes and 32,498 unigenes were also annotated in the Swanshot and eggNOG databases, respectively (Table 4). According to NCBI NR database and E-value distribution, the number of unigenes annotated in our dataset was 35,024 , of which $71.53 \%$ of these unigenes $\left(E<10^{-50}\right)$ had strong homology and $47.87 \%$ of these unigenes $\left(\mathrm{E}<10^{-100}\right)$ had very strong homology (Fig. 2a).

Ten popular-related species were also annotated based on the NCBI NR database (Fig. 2b). The highest homology to Acer pseudo-sieboldianum was Citrus sinensis, accounting for $12.25 \%$ homology, followed by Citrus clementina, which accounted for $9.74 \%$ homology.

The COG (Clusters of Orthologous Groups) database is a database for homologous classification of gene products. It is an early database used for the identification of directly homologous genes. It is based on a large number of comparisons of protein sequences from various organisms. 12,984 unigenes from our dataset were classified into 24 protein families, of which 1,867 unigenes (12.67\%) were classified as general function predictions only, followed by carbohydrate transport and metabolism, with 1,380 unigenes $(9.36 \%)$. The smallest number of identified unigenes was in extracellular structures, with only 15 unigenes $(0.1 \%)$. Also, a large number of unigenes were assigned to translation, ribosomal structure and biogenesis (9.26\%), posttranslational modification, protein turnover, chaperones $(8.09 \%)$, lipid transport and metabolism $(7.4 \%)$, energy production and conversion (6.68\%), amino acid transport and metabolism $(6.41 \%)$ and signal transduction mechanisms (6.26\%) (Fig. 3).

GO (Gene Ontology) databases are divided into three categories: Biological Process, Cellular Component and Molecular Function, which are further divided into 42 
functional subgroups. In Biological Process, which had the largest number of annotated unigenes, included metabolic process and cellular process with 13,141 (51.78\%) unigenes and 11,546 (45.5\%) unigenes, respectively. The Cellular Component class mainly included cell and cell part, with 11,886 (46.84\%) unigenes and 11,806 (46.53\%) unigenes, respectively. The Molecular Function category mainly included catalytic activity and binding, and there were 12,691 (50.01\%) unigenes and 1,1049 (43.54\%) unigenes in these two groups (File. S3).

These KEGG database annotated 12,487 unigenes assigned to 130 different KEGG pathways. Our results showed that the largest pathway group was Ribosome, which contained 832 (11.32\%) unigenes, followed by Carbon metabolism and Biosynthesis of amino acids, which contained 661 (8.99\%) and 487(6.63\%) unigenes, respectively.

Metabolic pathways directly related to plant leaf color and leaf growth (number of unigenes annotated) were Flavonoid biosynthesis (ko00941 28 unigenes), Phenylalanine metabolism (ko00360 74 unigenes), Anthocyanin biosynthesis (ko00942 1 unigene), and Porphyrin and chlorophyll metabolism (ko00860 73 unigenes), etc. (File. S4).

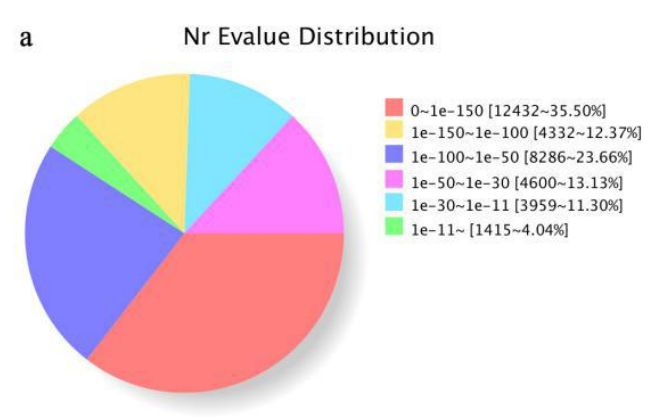

b

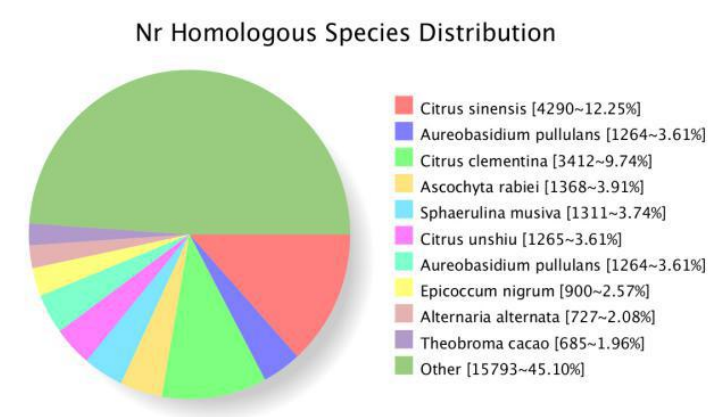

Fig. 2 Characteristics of homology search of Acer pseudo-sieboldianum unigenes. (a) E-value distribution in the NR database for each unigene. (b) Species taxonomy based on the NR database.

Table 4 Statistics of comparisons with databases

\begin{tabular}{|c|c|c|c|c|c|c|c|c|c|}
\hline Anno Database & $\mathrm{COG}$ & GO & KEGG & KOG & Pfam & Swisspro & eggNOG & $\mathrm{Nr}$ & All \\
\hline $\begin{array}{l}300<=\text { length }<1 \\
00\end{array}$ & 4534 & 11027 & 4861 & 7536 & 9281 & 6715 & 14214 & 16192 & 16431 \\
\hline length $>=1000$ & 8450 & 14348 & 7626 & 11924 & 15945 & 13081 & 18284 & 18832 & 18885 \\
\hline $\begin{array}{l}\text { annotated } \\
\text { number }\end{array}$ & 12984 & 25375 & 12487 & 19460 & 25226 & 19796 & 32498 & 35024 & 35316 \\
\hline
\end{tabular}




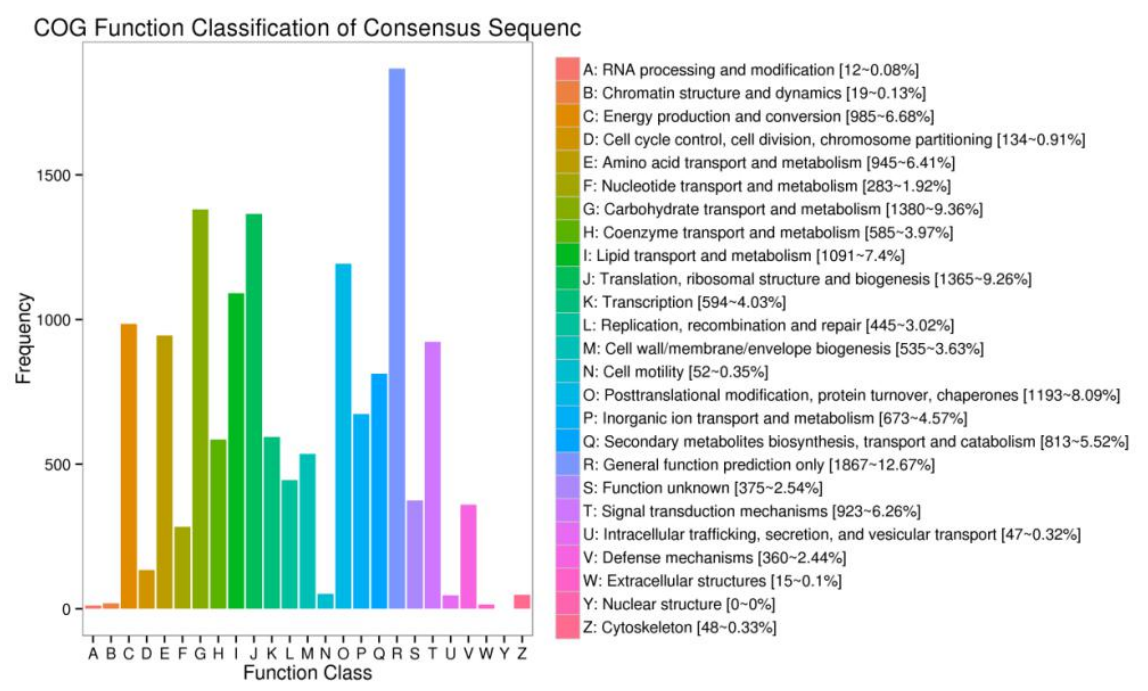

Fig.3 COG classifications of annotated unigenes

\section{Differentially expressed genes (DEGs)}

242 In order to explore the genes related to anthocyanin biosynthesis in Acer 243 pseudo-sieboldianum at different color-changing stages, the differential expression of 244 Acer pseudo-sieboldianum samples at different color-changing stages were then analyzed. The results showed that there were 16,521 DEGs in the three color-changing periods of Acer pseudo-sieboldianum. Comparing between the early stage (B) and the middle stage (M), there were 87 significant DEGs, with 52 up-regulated and 35 down-regulated. Between with the early stage (B) and the final stage (A), there were 14,855 DEGs, of which 7984 were up-regulated and 6871 were down-regulated. In a comparison of the middle stage (M) and the final stage (A), there were 12,402 DEGs, 5683 up-regulated and 6719 down-regulated, in Acer pseudo-sieboldianum (Figs. 4 and 5). In order to further understand the function of these respective DEGs, we carried out KEGG pathway enrichment analysis in the three stages of Acer pseudo-sieboldianum. Our results showed that there were 16,521 differentially expressed genes in the three stages (B, M and A). The flavonoid biosynthesis pathways related to leaf tone control were significantly enriched in B vs $\mathrm{M}$ and $\mathrm{B}$ vs A up-regulated genes. Phenylalanine metabolic pathways were significantly enriched in B vs M and B vs A up-regulated genes.

258 In addition, photosynthesis and photosynthesis-antenna protein pathways were 259 significantly down-regulated in B vs A (File. S5-S7). 


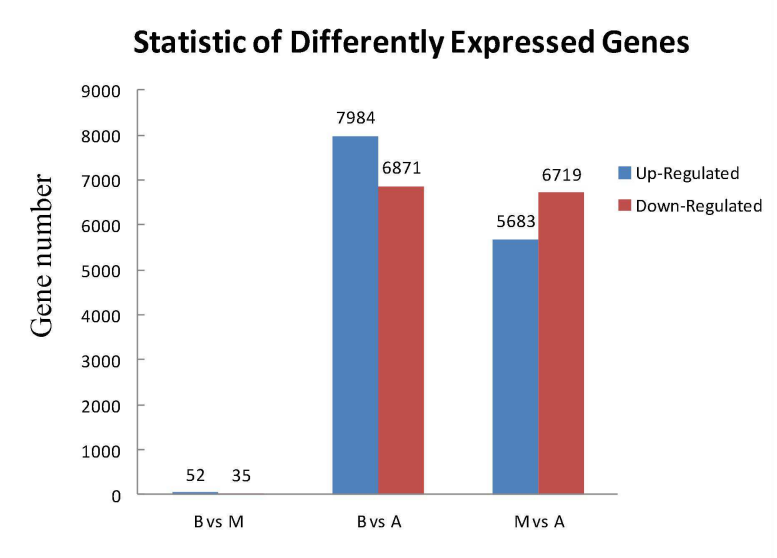

Fig. 4 Differentially expressed genes in the three stages

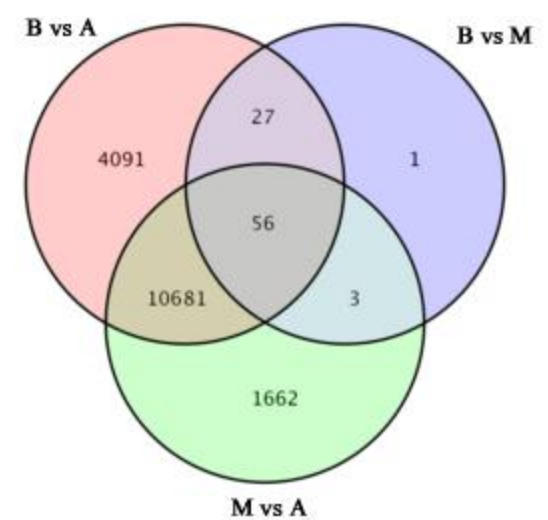

Fig. 5 Relationship of differentially expressed genes across development time.

Candidate genes involved in the flavonoid-anthocyanin biosynthesis

Pathway

Flavonoids are important secondary metabolites in plants. The pathway for flavonoid

biosynthesis has been well verified. We identified 20 candidate genes that covered almost all known enzymes involved in flavonoid biosynthesis (Fig. 6). Four PAL genes were detected in the upstream phenylalanine pathway (c118011.graph_c0, c118229.graph_c0, c60818.graph_c0, c97964.graph_c0), one CHS gene was detected (c100615.graph_c0),

271 one CHI gene (c108255.graph_c0), two F3H genes (c114916.graph_c0, 272 c56266.graph_c0), one F3'5'H gene (c111360.graph_c0), and two F3'H genes (c110935.graph_c0, c108910.graph_c0) were also detected. Among the four PAL genes,

274 the expression of a single gene, c118011.graph_c0, was significantly up-regulated in M vs A, but not in B vs M. The expression of the gene c118229.graph_c0 decreased in B vs M, but not in M vs A. Additionally, the expression of the genes c60818.graph_c0 and 
c97964.graph_c0 were up-regulated in B vs A . The expression of the single gene c100615.graph_c0 was significantly down-regulated in $\mathrm{M}$ vs $\mathrm{A}$. In contrast, the expression of the gene c108255.graph_c0 was down-regulated in $\mathrm{M}$ vs A, but not significantly in B vs M. The expression of the single gene c114916.graph_c0 increased in B vs $M$, but decreased significantly in M vs A. The expression of c111360.graph_c0 decreased significantly in M vs A, while the expression of c110935.graph_c0 increased significantly during the B vs M period.

One ANS gene (c60813.graph_c0) was found in the anthocyanin pathway branch, and its expression increased during B vs M. Two genes (c107656.graph_c0, c99050.graph_c0) encoding DFR were identified, and the expression of the gene c107656.graph_c0 increased significantly during B vs M. In the final modification stage of the anthocyanin pathway, six UFGT genes were identified, of which the expression of the single gene c103768.graph_c0 increased significantly during the B vs M period, while the expression of c120331.graph_c0 did not differ significantly during B vs M, while the expression of both genes decreased significantly during the $M$ vs $A$ period. The UFGT gene (c103768.graph_c0) increased significantly in the $B$ vs $M$ period, and decreased significantly in M vs A, which indicated that UFGT was first highly accumulated and then rapidly consumed. Combined with the detection and quantification of metabolites, paeoniflorin may be the most important anthocyanin species that caused the color to change to red in Acer pseudo-sieboldianum leaves. 


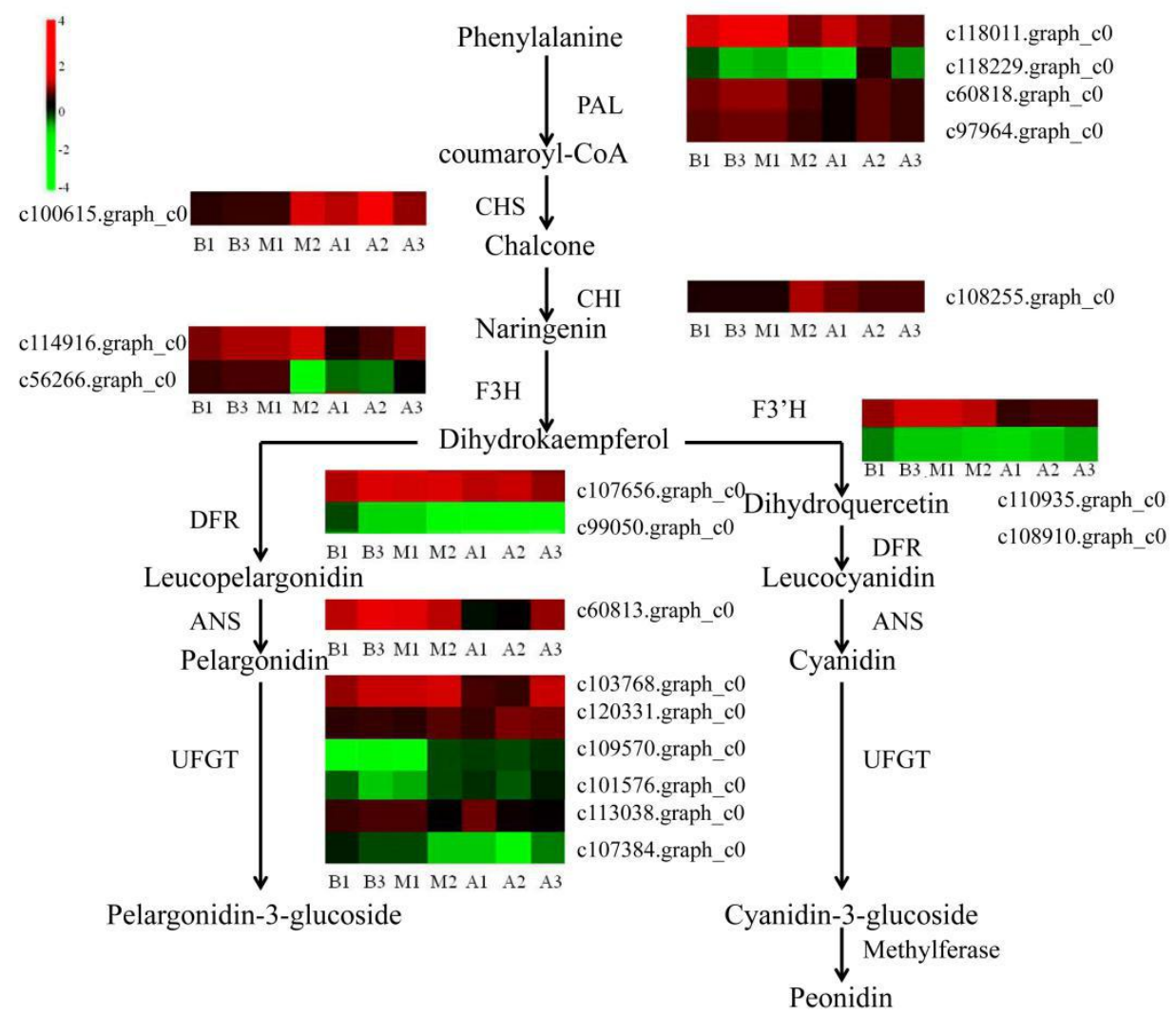

298 Fig. 6 Thermographic analysis of gene pathways related to flavonoid synthesis in Acer

299 pseudo-sieboldianum leaves at three stages. B, M and A are arranged horizontally at all

300 stages, and single genes are listed vertically. The annotations are displayed next to the

301 corresponding genes. All FPKM values of single genes are plotted logarithmically.

302 qRT-PCR confirmation of RNA-seq data

303 In order to verify the accuracy of our sequencing data, we selected eight genes involved

304 in flavonoid anthocyanin biosynthesis, and analyzed the expression level in leaves of 305 different color from these three different stages of Acer pseudo-sieboldianum by 306 qRT-PCR (Fig. 7). The results showed that these gene expression levels were consistent with our RNA sequencing data, demonstrating the reliability of our data. 
A c103768.graph_c0 B c107656.graph_c0 308
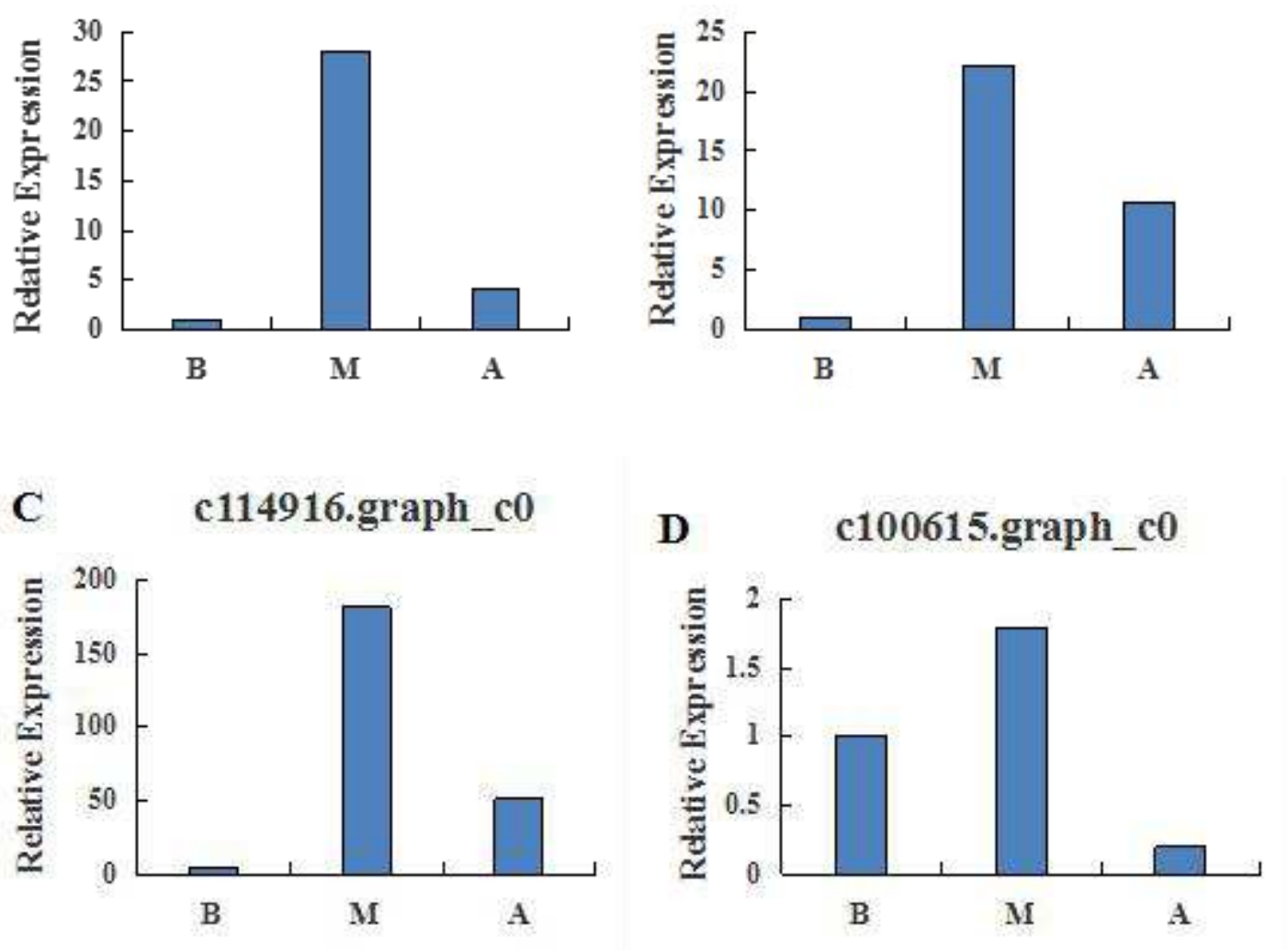

309
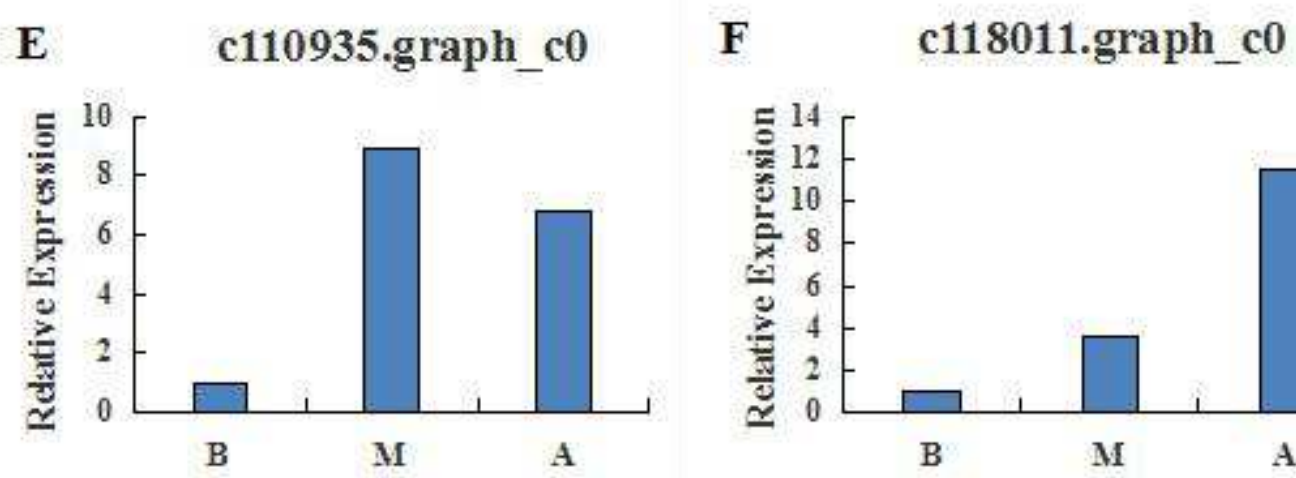

310
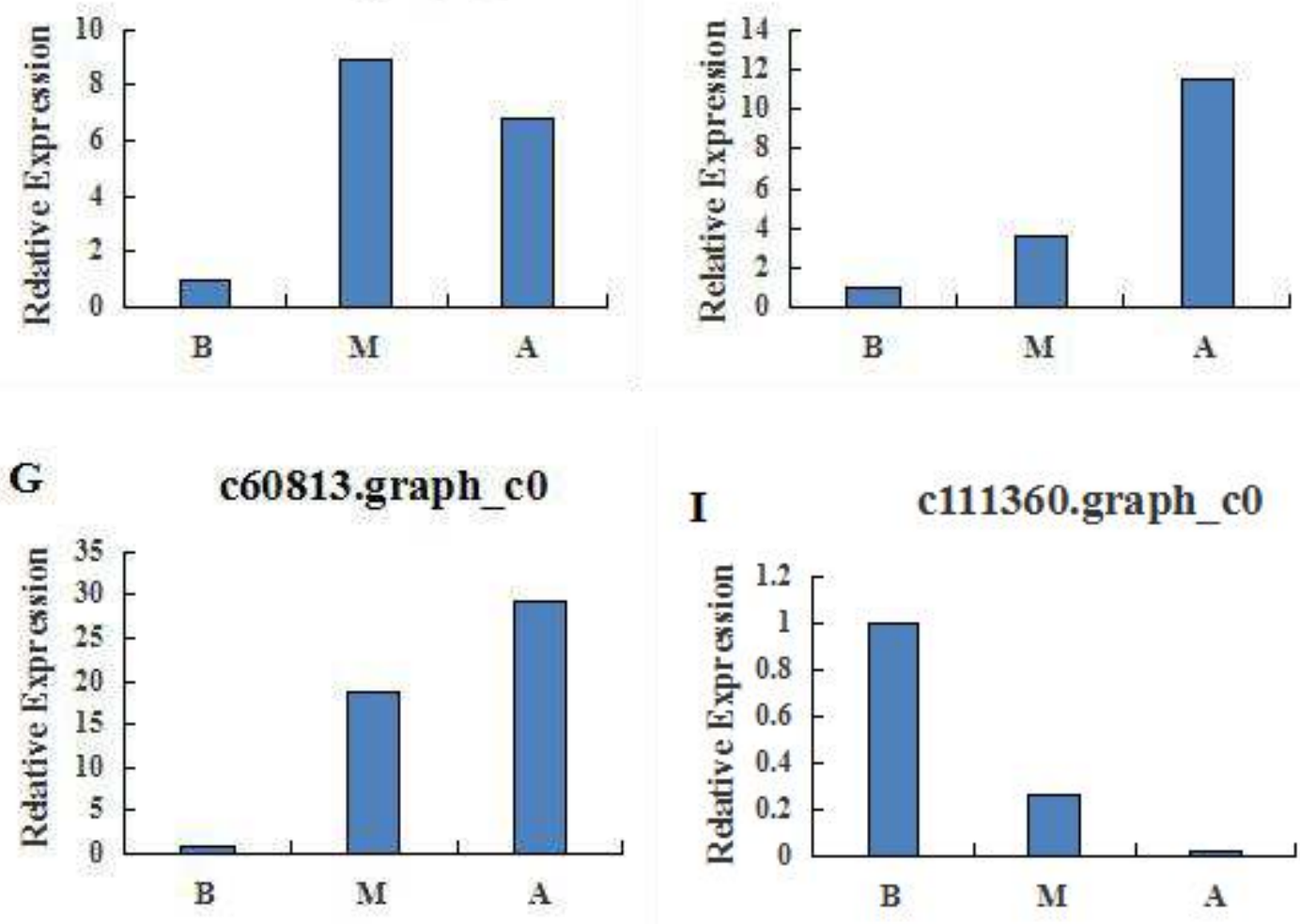

312 Fig. 7 Expression analysis of eight differentially expressed genes related to flavonoid and 
anthocyanin biosynthesis in Acer pseudo-sieboldianum.

\section{Discussion}

Acer pseudo-sieboldianum is an important ornamental plant in China. Its leaves are attractive in autumn, beautiful in shape, shining like the morning sun and flowers. Both Acer pseudo-sieboldianum and Acer palmatum Thunb. belong to Sect. Palmata Paxand Ser. Palmata (Pax) Pojark. Acer palmatum Thunb. has many varieties, gorgeous leaves, fast growth and strong ecological adaptability [41]. However, the distribution of Acer pseudo-sieboldianum is limited, and its growth potential is relatively slow. Therefore, most Acer pseudo-sieboldianum are still in a wild state and have not been widely used in gardens. Some scholars have analyzed the color mechanism of the related species in Acer rubrum L. [42].

At present, transcriptome sequencing technology has been used to study leaf color formation [43], flower color mechanisms[44, 45], fruit development [46, 47], and so on. However, due to the lack of genomic reference sequences, the molecular mechanism of leaf color is difficult to decipher in Acer pseudo-sieboldianum. The change of anthocyanin content in plants was shown to be related to the differential expression of key genes encoding structural enzymes in the anthocyanin biosynthesis pathway [48]. Jiang HB [49]screened the different genes in the flavonoid biosynthesis pathway from the purple bud tea plant by transcriptome sequencing, and there were nine enzyme genes, including PAL, CHS, ANS, UFGT, FLS, C4H, 4CL, DFR, and ANR, that were identified. In this study, we used transcriptome sequencing technology to sequence and compare three different coloring stages of Acer pseudo-sieboldianum leaves in autumn. We detected four PAL, one CHS, one CHI, two F3H, two F3'H, one F3'5'H, two DFR, one ANS, and six UFGT genes in the flavonoid anthocyanin complex related to leaf color in Acer pseudo-sieboldianum. Three of the UFGT single genes were down regulated in the M vs A stage, which indicated that the change of leaves from green to red was controlled by multiple single genes.

Both F3' $\mathrm{H}$ and F3'5' $\mathrm{H}$ belong to the cytochrome $\mathrm{P} 450$ superfamily [50]. F3' $\mathrm{H}$ is an important intermediate in the synthesis of cyanidin. F3' 5 ' $\mathrm{H}$ is a key enzyme in the 
synthesis of blue flower anthocyanin. Masukawa T [51] found F3'H could make red cyanidin accumulate in purple and red root radishes. Wu Q [52] found that F3'5'h mainly accumulated in the blue waterlily. Many important flower crops can't produce turquoise, meaning they cannot appear blue. In this study, we detected two F3'H and one $\mathrm{F}^{\prime} 5^{\prime} \mathrm{H}$, but the content of $\mathrm{F}^{\prime} 5^{\prime} \mathrm{H}$ in the $\mathrm{M}$ vs A period was very small and there was no significant difference, so Acer pseudo-sieboldianum leaves did not appear blue at this time.

DFR is the key enzyme that catalyzes the conversion of dihydroflavonol to corresponding colorless geranium, delphinium and cyanidin [53]. The main function of ANS is to oxidize colorless proanthocyanidins to produce colored anthocyanidins, which are the first colored compound in the anthocyanin synthesis pathway [54]. ANS was originally identified in a maize A2 mutant and cloned by the transposon tagging technique [55]. UFGT is mainly responsible for transforming unstable anthocyanins into stable anthocyanins. Studies have shown that the expression of UFGT is different in different varieties [56]. For example, anthocyanin accumulation in apple was positively correlated with UFGT activity. The change of UFGT activity in grape leads to the change of their phenotype from white to red [57]. In this study, UFGT (c103768.graph_c0) expression in Acer pseudo-sieboldianum leaves first accumulated and then was consumed in the process of leaf color formation, which was consistent with the conclusion that UFGT consumption was needed for paeoniae anthocyanin synthesis.

It is worth mentioning that we found Rosinidin O-hexoside in five anthocyanin components, although the content was very small. There was no Rosinidin O-hexoside found in previous studies on anthocyanins of Acer. Schmitzer V [58] found that the content of cyanidin 3-glucoside could be used as a quantitative index to determine the position effect of Acer palmatum atropurpureum. Cai XY [59] found that the reason leaf color changed from red to green in Acer palmatum Thunb. was the result of the increase of the mass fraction of cyanidin galactoside and the decrease of the mass fraction of chlorophyll. The distribution of Rosinidin O-hexoside in plants is very limited and has only been reported in Catharanthus roseus [60] and Primula [61]. 
The contents of differential metabolites were very high in terms of Peonidin O-hexoside, Cyanidin 3-O-glucoside (Kuromanin) and Cyanidin 3,5-O-diglucoside (Cyanin). These results demonstrate a molecular mechanism of leaf color change in autumn in Acer pseudo-sieboldianum and provide the basis for molecular breeding theory for ornamental plant leaf color improvement.

\section{Conclusions}

In this study, we detected five anthocyanins in the leaves of Acer pseudo-sieboldianum, including Rosinidin O-hexposite, the distribution of which in Acer plants is very limited. We used the transcriptome data obtained using the high-throughput sequencing technology to provide an effective method for the identification of three important single genes in autumn leaves of Acer pseudo-sieboldianum. A total of 50,501 unigenes were produced in three stages of Acer pseudo-sieboldianum leaves, among which 16,521 DEGs and 64 unigenes were identified as color-related homologous genes. In this study, we identified anthocyanins related to the anthocyanin synthesis pathway and red leaf formation. We detected four PAL, one CHS, one CHI, two F3H, two F3'H, one F3'5'H, two DFR, one ANS, and six UFGT in the flavonoid anthocyanin complex related to leaf color in Acer pseudo-sieboldianum. Then, UFGT was selected as the most important candidate compound to participate in the anthocyanin metabolism pathway and induce red leaf formation. This study provides a theoretical basis for the formation of leaf color in Acer pseudo-sieboldianum.

\section{Additional files}

Additional files 1: Primer Information in Acer pseudo-sieboldianum. (DOCX $14 \mathrm{~kb}$ )

Additional files 2: Anthocyanins detected in Acer pseudo-sieboldianum. (XLSX $1565 \mathrm{~kb}$ )

Additional files 3: Histogram of GO classification of assembled unigenes. (DOCX $160 \mathrm{~kb})$

Additional files 4: KEGG pathway annotation in Acer pseudo-sieboldianum unigenes. (XLSX $15 \mathrm{~kb}$ )

Additional files 5: KEGG pathway enrichment analysis of DEGs between B and A. (XLSX $22 \mathrm{~kb}$ )

Additional files 6: KEGG pathway enrichment analysis of DEGs between B and M. (XLSX $13 \mathrm{~kb}$ )

Additional files 7: Analysis map of gene pathway for differential expression of 
flavonoid biosynthesis pathway. Map of differentially expressed gene pathways in phenylalanine metabolism.(DOCX $96 \mathrm{~kb}$ )

\section{Abbreviations}

RNA-Seq: RNA sequencing; RT-qPCR: Real-time quantitative polymerase chain reaction; Q30 percentage: Percentage of bases with sequencingerror rate lower than $1 \%$; NR: Non-redundant; Pfam: Protein family; DEGs: Differentially expressed genes; COG: Clusters of Orthologous Groups of proteins; GO: Gene Ontology; KEGG: Kyoto Encyclopedia of Genes and Genomes; KOG: Eukaryotic Orthologous Groups of proteins; ANS: Anthocyanidin synthase; CHI: Chalcone isomerase; CHS: Chalcone synthase;DFR:Dihydroflavonol 4-reductase;F3H: Flavanone 3-hydroxylase;F3'5'H: Flavonoid 3', 5'-hydroxylase; F3'H: Flavonoid 3'-hydroxylase;UA3GT: UDP-glycoside:Anthocyanin 3-O-glycosyltransferase; UFGT:UDP-glycoside: flavonoid 3-O-glucosyl-transferase.

\section{Funding}

The project was supported by Science and Technology Development Plan Project of Jilin Province (20180520218JH).

\section{Availability of data and materials}

The datasets supporting the conclusions of this article are included within the article and its additional files.

\section{Authors' contributions}

DZ and YG contributed equally to this work. DZ and YG carried out sequence data analysis and drafted the manuscript. LR organized the manuscript and supervised the study. JZ and JC collected taproot samples. All authors have read, revised the manuscript critically for important intellectual content, and ap proved the final manuscript. All authors agreed to be accountable for all aspects of the work in ensuring that questions related to the accuracy or integrity of the manuscript are appropriately investigated and resolved.

\section{Ethics approval and consent to participate}

Not applicable.

\section{Consent for publication}

Not applicable.

\section{Competing interests}

The authors declare that they have no competing interests. 
${ }^{1}$ Agriculture college, Yanbian University, Yanji 133002, China

[1] Chalker SL. Environmental significance of anthocyanins in plant stress responses.

Photochemistry and Photobiology. 1999;70:1-9. DOI:10.1111/j.1751-1097.1999.tb01944.x

[2] Dai SL, Huang H, Fu JX, Hong Y. Advances in molecular breeding of ornamental plants.Chinese

[3] Li XM. Physiological characters of Pyracantha fortuneana 'Harlequin' leaves during color-changing period in autumn and winter. Journal of Shanghai Jiaotong University(Agricultural Science). 2013;31:82-87. DOI:10.3969/J.

[4] Ren J, Chen Z, Tang F, Xuan Y, Yang F, Lu XY, Fu SL. Study on leaf color related chemicals components based on comparing Acer rubum L. cv. 'Yanhong' and 'Jinseqiutian'. Journal of Anhui Agricultural University. 2019;46:420-425. DOI:10.13610/j. serrata leaves during leaf color transformation in autumn. Scientia Silvae Sinicae. 2015;51:44-51. DOI:10.11707/j.1001-7488.20150806 ident. Sin. 2019;39:808-816. DOI:10.7606/j.

464 [10] Jin XH. The study of anthocyanidins biosynthesis pathway in Senecio cruentus 
[11] Wang W, Zheng W, Xu XD, Chen J, Wang TX. Coloring mechanism analysis of mosaic le aves in Camellia reticulata Lindl. based on sequencing of transcriptome.Acta Bot. Boreal. Occid ent. Sin. 2017;37:1720-1727. DOI:10.7606/j.

[12] Jia ZD, Ma PY, Bian XF, Yang Q, Guo XD, Xie YZ. Biosynthesis metabolic pathway and molecular regulation of plants anthocyanin. Acta Bot. Boreal. Occident. Sin. 2014;34:1496-1506. DOI:10.7606/j.

[13] Liu XF, Li F, Yin XR, Xu CJ, Chen KS. Recent advances in the transcriptional regulation of anthocyanin biosynthesis. Acta Horticulturae Sinica. 2013;40:2295-2306. DOI:10.16420/j.

[14] Duan YJ, Zhang LG, He Q, Zhang MK, Shi JC. Expression of transcriptional factors and structural genes of anthocyanin biosynthesis in Purple-heading Chinese Cabbage. Acta $\mathrm{H}$ orticulturae Sinica. 2012;39:2159-2167. DOI:10.16420/j.

[15] Zhu JL. Physiological characteristics of leaf color establishment and key gene screening of Eucommia ulmoides 'Hongye'. Chinese Academy of Forestry.2017.

[16] Grace SC, Logan BA, Adams WW. Seasonal differences in foliar content of chlorogenic a cid, A phenylpropanoid antioxidant, in mahonia repens. Plant, Cell \& Environment, 1998;21:513521. DOI:10.1046/j.1365-3040.1998.00282.x

[17] Zhang X, Xiao TT, Li J, Wang YT, Liu GL. Effects of water stress on the growth and leaf color in Acer rubrum. Jiangsu Agricultural Sciences, 2016;44:224-227. DOI:10.15889/j.

[18] Oberbauer SF, Starr G. The role of anthocyanins for photosynthesis of alaskan arctic everg reens during snowmelt. Advances in Botanical Research. 2002;37:129-145. DOI:10.1016/S0065-22 $96(02) 37047-2$

[19] Sun B, Zheng DC, Cui HM. Leaf color change in Acer pseudo-sieboldianum in autumn.Journal of Northeast Forestry University. 2009;37:14-15. DOI:CNKI:SUN:DBLY.0.2009-02-007

[20] Pang QY, Zhuo LH. Spatial difference in physiological indexes of autumn colored Maple leaves. Journal of Northeast Forestry University. 2007;35:16-17,20. DOI:10.13759/j.

[21] Xian Y, Dong X, Xie XM, Wu D, Han B, Wang Y. Effect of conservation conditions on restricting conservation of Acer rubrum cv. 'Somerset'. Chinese Bulletin of Botany. 2019;54:64-71. DOI:10.11983/CBB 18010

[22] Qu X, Sun B, Yang YH. Leaf color performance and its application of introduced Acer pseudo-sieboldianum. Terrttory \& Natural Resources Study. 2012;:86-88. DOI:10.16202/j. 
[23] Zhao LY, Cao SY, Long YS, Zhang YH, Xu JQ. Applications and research progresses of $\mathrm{f}$ ull-length transcriptome sequencing in plants. Journal of Plant Genetic Resources. 2019;20:1-12. DOI: $10.13430 / j$.

499

[24] Wang YS, Wang H, Fan ZY, Chen Y, Jin YF, Gao ML. Identifying genes associated with leaf color in kale (Brassica oleracea L. var. acephala DC.) based on transcriptome analysis. Genomics and Applied Biology. 2019;38:1-9. http://kns.cnki.net/kcms/detail/45.1369.Q.20190422.1420.006.html [25] Kanehisa M, Goto S, Kawashima S, Okuno Y, Hattori M. The KEGG resource for deciphe ringthe genome. Nucleic Acids Research. 2004;32:277-280. DOI: 10.1093/nar/gkh063 Raychowdhury R, Zeng QD, Chen ZH, Mauceli E, Hacohen N, Gnirke A, Rhind N, Dipalma F, Birren BW, Nusbaum C, Lindblad-Toh K, Friedman N, Regev A. Full length transcriptome a ssembly from RNA Seq data without a reference genome. Nature Biotechnology. 2011;29:644-65 2. DOI: $10.1038 /$ nbt. 1883

[27] Deng YY, Li JQ, Wu SF, Zhu YP, Chen YW, He FC. Integrated nr database in protein an notation system and its localization. Computer Engineering. 2006; 32:71-74. DOI:10.1109/INFOC

[28] Apweiler R, Bairoch A, Wu CH, Barker WC, Boeckmann B, Ferro S, Gasteiger E, Huang rot: the Universal Protein knowledgebase. Nucleic Acids Research. 2004;32:115-9. DOI: 10.1093/nar/gkh131

521 [30] Tatusov RL, Galperin MY, Natale DA, Koonin EV. The COG database: a tool for genome

[29] Ashburner M, Ball CA, Blake JA, Botstein D, Butler H, Cherry JM, Davis AP, Dolinski K, scale analysis of protein functions and evolution. Nucleic Acids Research. 2000;28:33-36. DOI: $10.1093 / \mathrm{nar} / 28.1 .33$

[31] Koonin EV, Fedorova ND, Jackson JD, Jacobs AR, Krylov DM, Makarova KS, Mazumber R, Mekhedov SL, Nikolskaya AN, Rao BS, Rogozin IB, Smirnow S, Sorokin AV, Sverdlov AV, 
Vasudevan S, Wolf YI, Yin JJ, Natale DA. A comprehensive evolutionary classification of prot eins encoded in complete eukaryotic genomes. Genome Biology. 2004;5:R7. DOI:10.1186/gb-200 $4-5-2-r 7$

[32] Huerta-Cepas J, Szklarczyk D, Forslund K, Cook H, Heller D, Walter MC, Ratteri T, Mende

DR, Sunagawa S, Kuhn M, Jensen LJ, Mering CV, Bork P. eggNOG 4.5: a hierarchical orthology framework with improved functional annotations for eukaryotic, prokaryoticand viral sequences. Nucleic Acids Research. 2016;44:286-293. DOI:10.1093/nar/gkv1248 [33] Altschul SF, Madden TL, Schaffer AA, Zhang J, Zhang Z, Miller W, Lipman DJ. Gapped BLAST and PSI BLAST:A newgeneration of protein database search programs. Nucleic Acids R esearch. 1997;25: 3389-3402. PMID:9254694

[34] Xie, C, Mao, XZ, Huang, JJ, Ding, Y, Wu, JM, Dong, S, Kong, L, Gao, G, Li CY, Wei, LP. KOBAS 2.0: a web server for annotation and identification of enriched pathways and diseas es. Nucleic Acids Research. 2011;39:316-322. DOI:10.1093/nar/gkr483 [35] Eddy SR. Profile hidden Markov models. Bioinformatics. 1998;14:755-763. DOI:10.1093/bio informatics/14.9.755 [36] Finn RD, Bateman A, Clements J, Coggill P, Eberhardt RY, Eddy SR, Heger A, Hetheringt on K, Holm L, Mistry J, Sonnhammer ELL, Tate J, Punta M. Pfam: the protein families databa se. Nucleic Acids Research. 2014;42:222-230. DOI:10.1093/nar/gkt1223

[37] Langmead B, Pop M. Ultrafast and memory-efficient alignment of short DNA sequences to the human genome. Genome Biology. 2009;10:R25. DOI:10.1186/gb-2009-10-3-r25

[38] Li B, Dewey CN. RSEM:accurate transcript quantification from RNA-Seq data with or wit hout a reference genome. BMC Bioinformatics. 2011;12:323 DOI:10.1186/1471-2105-12-323 [39] Trapnell C, Williams BA, Pertea G, Mortazavi A, Kwan G, van Baren MJ, Salzberg SL,

Wold BJ, Pachter L. Transcript assembly and quantification by RNA Seq reveals unannotated tra nscripts and isoform switchingduring cell differentiation. Nature Biotechnology. 2010;28:511-515. DOI:10.1038/nbt.1621

[40] Pfaffl MW. A new mathematical model for relative quantification in real-time RT-PCR. Nuc leic Acids Research. 2001;29:e45. DOI:10.1093/nar/29.9.e45

[41] Rong LP, Li QZ, Li SS, Tang L, Wen J. De novo transcriptome sequencing of Acer palma tum and comprehensive analysis of differentially expressed genes under salt stress in two contra 
sting genotypes. Molecular Genetics and Genomics. 2016;291:575-586. DOI:10.1007/s00438-015-1 $127-2$

[42] Chen Z, Lu XY, Xuan Y, Tang F, Wang JJ, Shi D, Fu SL, Ren J. Transcriptome analysis based on a combination of sequencing platforms provides insights into leaf pigmentation in Acer rubrum. BMC Plant Biology. 2019;19:240. DOI:10.1186/s12870-019-1850-7

[43] Fang Z, Zhou DR, Ye XF, Jiang CCi , Pan SL. Identification of candidate anthocyanin-related genes by transcriptomic analysis of 'Furongli' Plum (Prunus salicina Lindl.) during fruit ripening using RNA-Seq. Front Plant Science. 2016;7:1338. DOI:10.3389/fpls.2016.01338

[44] Qu Y, Ou Z, Yang FS, Wang S, Peng JS. The study of transcriptome sequencing for flowe $\mathrm{r}$ coloration in different anthesis stages of alpine ornamental herb (Meconopsis 'Lingholm'). Gen e. 2018;689:220-226. DOI:10.1016/j.gene.2018.12.017

[45] Zhang HS, Tian H, Chen MX, Xiong JB, Cai H, Liu Y. Transcriptome analysis reveals pot ential genes involved in flower pigmentation in a red-flowered mutant of white clover (Trifolium repens L.). Genomics. 2018;110:191-200. DOI:10.1016/j.ygeno.2017.09.011

[46] Kodama M, Brinch-Pedersen H, Sharma S, Holme IB, Joernsgaard B, Dzhanfezova T, Amby DB, Vieira FG, Liu SL, Gilbert MTP. Identification of transcription factor genes involved in anthocyanin biosynthesis in carrot (Daucus carota L.) using RNA-Seq. BMC genomics, 2018,19:811. DOI: $10.1186 / \mathrm{s} 12864-018-5135-6$

[47] Shi LY, Chen X, Chen W, Zheng YH, Yang ZF. Comparative transcriptomic analysis of white and red Chinese bayberry ( Myrica rubra) fruits reveals flavonoid biosynthesis regulation. Scientia Horticulturae. 2018,235:9-20. DOI:10.1016/j.scienta.2018.02.076

[48] Zhuang HM, Lou Q, Liu HF, Han HW, Wang Q, Tang ZH, Ma YM, Wang H. Differential regulation of anthocyanins in green and purple turnips revealed by combined de novo transcrip tome and metabolome analysis. International Journal of Molecular Sciences. 2019;20:4387. DOI: $10.3390 / \mathrm{ijms} 20184387$

[49] Jiang HB, Xia LF, Tian YP, Dai WD, Sun YN, Chen LB. Transcriptome analysis of antho cyanin synthesis related genes in purple bud tea plant. Journal of Plant Genetic Resources. 2018; 19:967-978. DOI:10.13430/j. 
[50] Rosati C, Cadic A, Duron M, Ingouff M, Simoneau P. Molecular characterization of the an thocyanidin synthase gene in Forsythis Xintermedia reveals organ-specific expression during flow er development. Plant Science. 1999;149:73-79. DOI:10.1016/s0168-9452(99)00146-6

[51] Masukawa T, Cheon KS, Mizuta D, Kadowaki M, Nakatsuka A, Kobayashi N. Developmen $\mathrm{t}$ of mutant $\mathrm{RsF}^{\prime} \mathrm{H}$ allele-based marker for selection of purple and red root in radish (Raphanu s sativus L.var.longipinnatus L.H.Bailey). Euphytica. 2019;215:1-9. DOI:10.1007/s10681-019-24421

[52] Wu Q, Wu J, Li SS, Zhang HJ, Feng CY, Yin DD, Wu RY, Wang LS. Transcriptome sequencing and metabolite analysis for revealing the blue flower formation in waterlily. BMC Genomics. 2016;17:897. DOI:10.1186/s12864-016-3226-9

[53] Holton TA, Cornish EC. Genetics and biochemistry of anthocyanin biosynthesis. The Plant Cell. 1995;7:1071-1083. DOI:10.2307/3870058

[54] Springob K, Nakajirna J, Yamazaki M, Saito K. Recent advances in the biosynthesis and a ccumulation of anthocyanins. Natural Product Reports. 2003;20:288-303. DOI:10.1039/b109542k [55] Menssen A, Hohmann S, Martin W, Schnable PS, Peterson PA, Saedler H, Gierl A. The E $\mathrm{n} / \mathrm{Spm}$ transposable element of Zea mays contains splice sites at the temini generating a novel $\mathrm{i}$ ntron from a dSpam element in the A2 gene. The EMBO Journal. 1990;9:3051-3057. DOI: 10.1002/j.1460-2075.1990.tb07501.x

[56] Li JC, Li TH, Wang ZG, Li BJ. Cloning and expression of UFGT gene in skin of max re d Bartlett. Acta Botanica Boreali-Occidentalia Sinica. 2010;30:30-34. DOI:10.3724/SP.J.1238.2010. 00512

[57] Kobayashi S, Ishimaru M, Ding CK, Yakushiji H, Goto N. Comparison of UDP-glucose:fla vonoid 3-O-glucosyltransferase(UFGT) gene sequences between white grapes (Vitis vinifera) and their sports with red skin. Plant Science. 2001;160:543-550. DOI:10.1016/s0168-9452(00)00425-8 [58] Schmitzer V, Stampar F, Veberic R, Osterc G. Phase change modifies anthocyanin synthesis in Acer palmatum Thunb. (Japanese maple) cultivars. Acta Physiologiae Plantarum. 2009;31:415 -418. DOI:10.1007/s11738-008-0237-4

[59] Cai XY, Li HH, Li L, Yu H, Chen G, Bao NNDT. Pigment composition and leaf color ch ange in Acer palmatum. Journal of Northeast Forestry University. 2015;43:63-67. DOI:10.13759/j. 
613 [60] Toki K, Saito N, Irie Y, Tatsuzawa F, Shigihara A, Honda T. 7-O-Methylated anthocyanidin 614 glycosides from Catharanthus roseus. Phytochemistry. 2008;69:1215-1219. DOI:10.1016/j.phytoch $615 \quad$ em.2007.11.005

616 [61] Valentina S, Maja MP, Franci S, Vlasta C. Phenolic accumulation in Hybrid Primrose and pigment 617 distribution in different flower segments. Journal of the American society for horticultural science.

$618 \quad 2017 ; 142: 192-199$. DOI:10.21273/JASHS04038-17

619

620

621 


\section{Supplementary Files}

This is a list of supplementary files associated with this preprint. Click to download.

- File.S5.xlsx

- File.S7.docx

- File.S2.xlsx

- File.S3.docx

- File.S6.xlsx

- File.S1.docx

- File.S4.xlsx 